\title{
The magnetic switch manufacturing by using ferrofluid and
}

\section{ferrofluid doped copper nanoparticles}

\author{
Rehab Abd Al Hussin Ali ${ }^{1}$, Hashim Ali Yusr ${ }^{1}$, Soudad S. Bassam ${ }^{2}$ \\ ${ }^{1}$ Department of Physics, College of Science, University of Wassat \\ ${ }^{2}$ Department of Physics, College of Science, University of Baghdad \\ Corresponding author: soudadbassam@gmail.com
}

\begin{abstract}
In this work, a magnetic switch was prepared using two types of ferrofluid materials, the pure ferrofluid and ferrofluid doped with copper nanoparticles (10 nm). The critical magnetic field (Hc) and the state of magnetic saturation (Hs) were studied using three types of laser sources. The main parameters of the magnetic switch measured using pure ferrofluid and He-Ne Laser source were Hc (0.5 mv, $0.4 \mathrm{G})$, Hs (8.5 mv, $3 \mathrm{G})$. For the ferrofluid doped with copper nanoparticles were Hc (1 mv, 4 G), Hs (15 mv, 9.6 G), Using green semiconductor laser for the Pure ferrofluid were Hc $(0.5 \mathrm{mv}$, $0.3 \mathrm{G}) \mathrm{Hs}(15 \mathrm{mv}, 2.9 \mathrm{G})$. While the ferrofluid doped with copper nanoparticles were Hc ( $0.5 \mathrm{mv}, 1 \mathrm{G})$, Hs (12 mv, $2.8 \mathrm{G})$ and by using the violet semiconductor laser the pure ferrofluid parameters were $\mathrm{Hc}$ ( $0.5 \mathrm{mv}, 0.5 \mathrm{G})$, Hs $(15 \mathrm{mv}, 3.5 \mathrm{G})$ while the ferrofluid doped with copper nanoparticles are Hc (0.5 mv, $1 \mathrm{G})$, Hs (15 mv, $3.3 \mathrm{G})$.
\end{abstract}

Key words

Magnetic switch, ferrofluid, copper nanoparticals, diode laser, He-Ne laser.

Article info.

Received: Dec. 2019

Accepted: Jun. 2020

Published: Sep. 2020
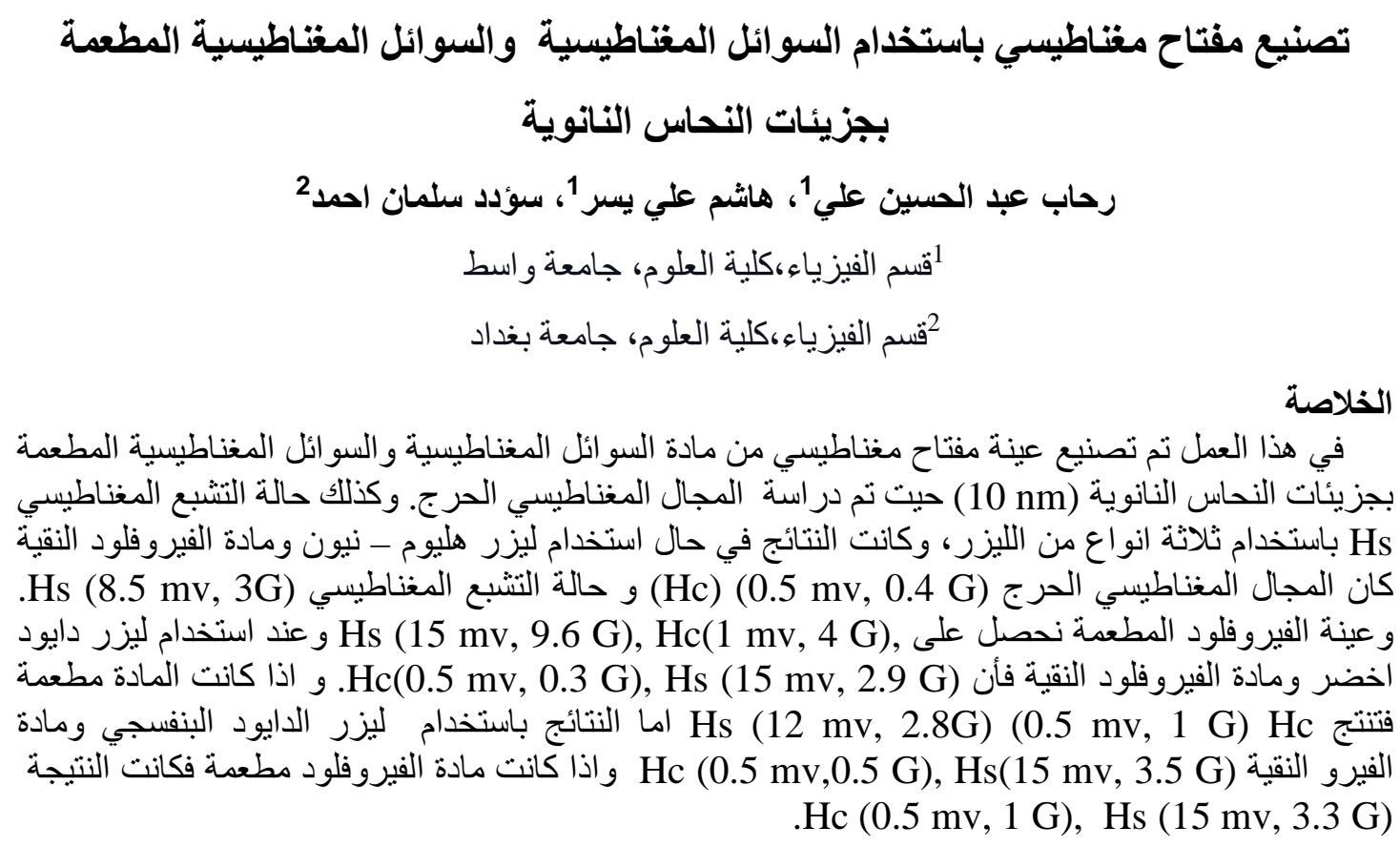

\section{Introduction}

Ferrofluids have novel characteristics and their behavior in magnetic fields results in many thrilling applications. Although magnetic fluids are already utilized in a few devices they have got now no longer but been exploited to any extent. 
Ferrofluids are magnetic liquids used in magnetic key manufacturing. They are stable colloidal dispersions of tiny (3-15 nm) super-paramagnetic particles coated with a surfactant. These particles remain suspended due to Brownian motion while the surfactant coating around each particle prevents the particles from sticking to each other. Ferrofluids become strongly magnetized upon the presence of a magnetic field and do not retain magnetization once the magnetic field is removed [1]. This magnetic fluid (ferrofluid) was first produced by Gowan Knight in 1779 from iron in water. However, his attempts were not successful because the particles deposited at the bottom of the new techniques of ferrofluid production have high accuracy. The ferrofluid particles are spread randomly (oil or water) [2]. Combine with nanotechnology, where the liquids such as water and oil are injected with very small particles (up to 10 million meters) of magnetic iron oxide, the injected particles are so small that they do not affect the liquidity of the liquid placed in it. As a result this fluid gains magnetic properties when placed in a magnetic field [3]. Ferrofluids can be formed in various forms, it can be applied to any type of industry, whether heavy industries or nanotechnology industries [4]. The human being researched, space and developing new and modern methods and techniques such as rocket engine work at the zero gravity, therefore NASA used kerosene-based magnetic fluid in the 1960s [5]. Ferrofluids a fascinating magnetic liquid used in many applications since the 1960s has pushed several researchers into carrying out studies on its potential in several applications. In 2014 Chin Wang and Luogen Deng, demonstrated a coherent, electrically controlled random laser with silver nanoparticles dispersed in an anesthetically dyeing liquid crystal (NLC) crystal, where the external emission intensity and frequency division were recorded. This provides evidence to support that the current laser resonance is very sensitive to buffer disturbances in the host medium, possibly associated with some oscillations associated with metallic nanoparticles [6]. ALMeshahedi in 2015 used 5CB as the liquid crystal to manufacture liquid crystal optical switch cell samples by employ the sandwich method [7]. In 2018, AL-Waali made optical switch using ferrofluid and ferromatic LC. He also studied the nonlinear optical properties [8]. In 2018, Youn-Jea Kim [9] studied the rotating Eccentric Cylinder by External Magnetic Force. They found that heat dissipation is an important problem because the volume of the electronic devices is small. The ferrofluids have a great role in the improvement of the electromagnetic systems. The ferrofluid is a colloidal solution consist of $10-\mathrm{nm}$ magnetic particles and showed paramagnetic behavior and very sensitive to temperature and magnetic field. The heat transferring of the ferrofluids is done into the rotating cylinder by using comsol multi physics. In 2018, Elena Castro-Hernández et al. [10], studied the ferrofluid is suspensions consist of magnetic nanoparticles in the liquid. The ferrofluid are used for research purposes in space (microgravity). The microgravity is an unexplored field because it is expensive and the access is limited. In 2018, Milorad M. Kuraica et al. [11] studied the magnetic field effect on the magnetite and cobalt-Fe NPS by simple optical measurements. The selective spectral behavior of suspended nanoparticles of magnetite $\left(\mathrm{Fe}_{3} \mathrm{O}_{4}\right)$ NPs and cobaltferrite $\left(\mathrm{CoFe}_{2} \mathrm{O}_{4}\right)$ coated with starch, was observed through switching on/off of the external magnetic field [11]. In 
2019, Huang et al., found that simulation of ferrofluids is similar to the simulation of the complex dynamics of ferrous, as the magnetic particles of ferrofluids react with an external magnetic field [12]. During this work design and implementation of magnetic switch based on ferrofluid and ferrofluid doped with copper nanoparticals is presented.

\section{Experimental work}

The first step was preparing the magnetic switch cell using indium tin oxide (ITO) coated glass slides (conductive layer) were coated with polyvinyl alcohol (PVA) as an insulating alignment layer. Electrical electrodes (Al) were deposited above the ITO glass substrate. After scratching the (PVA) layer by a piece of clothes in one direction, the mailer spacer of the small square-shaped mask was fixed on one of the plates. The ferrofluid was placed in the small square-shaped mask, then the two ITO glasses electrodes were fixed together with clips or adhesive material to form the final cell as shown in Fig.1.

Fig.1: The prepared sample of magnetic switch.

The doped ferrofluid sample was made using (0.012 mg) copper nanoparticles mixed with water-based ferrofluid. From the above steps, the doped ferrofluid with copper nanoparticles is obtained. The second step was studying the magnetic switch properties using the setup shown in Fig.2 the experimental setup consists of, a coil that was made with thick wire to reduce the heating effects (the coil properties such as type of wire (copper), the value of the current (1A), diameter of wire $(1 \mathrm{~mm})$, number of turns (6500), magnetic field (200 mT) have to be considered), Polarizer and analyzer (from Spindler and Hoyer Gottingen made in Japan), DC power supply (made in Germany
(QJ3005X ш)) which supplies (9V) DC voltage to the coil, Power meter made (by Mobiken), laser power meter (LP1made in Germany), and photodiode $(\lambda(400-1100 \mathrm{~nm})$, measuring range power $(40 \mu \mathrm{w}-$ $40 \mathrm{MW})$ ), accuracy ( $\pm 5 \%$ (4mw, $\lambda=632 \mathrm{~nm})$, Different lasers including: He-Ne Laser (2mW, 635.8nm), which is a light source used to characterize the optical transmissions of the samples; green semiconductor laser $(\lambda=532 \mathrm{~nm}, 30 \mathrm{~mW})$; violet semiconductor laser $(\lambda=405 \mathrm{~nm}$, $5 \mathrm{~mW}$ ), Gauss meter (supplies voltage $(9 \mathrm{~V})$, made in China) and magnetic switch cell placed between a pair of perpendicular polarizer and analyzer as shown in Fig.2. 


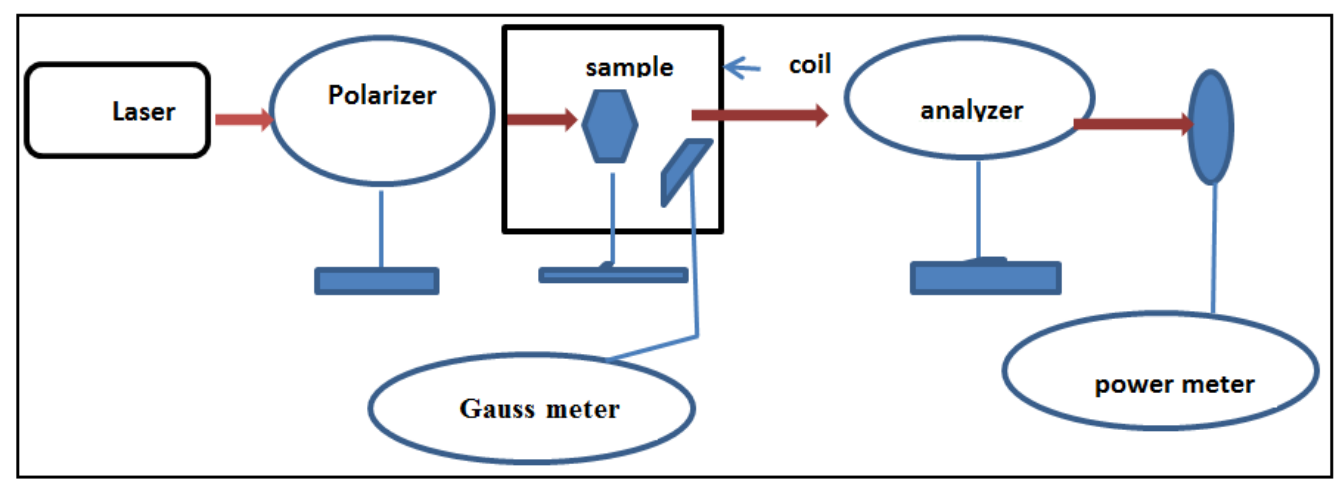

Fig.2: Schematic diagram of setup of magnetic switch.

\section{Results and discussion}

By radiating the samples with He$\mathrm{Ne}$, green semiconductor laser and violet semiconductor laser the critical magnetic field, the saturation magnetic field, and the hysteresis loop were obtained. The critical and the saturation magnetic fields values for the pure and doped ferrofluid samples using the three types of laser source are shown in Table 1.

Table 1: The critical and the saturation magnetic field of the samples.

\begin{tabular}{|c|c|c|c|}
\hline Laser & Material & Hc (Gauss) & Hs (Gauss) \\
\hline \multirow[b]{2}{*}{ Violet semiconductor laser } & ferrofluid Pure & $0.5-0.5$ & $15-3.5$ \\
\hline & $\begin{array}{l}\text { ferrofluid doped } \\
\text { with copper } \\
\text { nanoparticles }\end{array}$ & $0.5-1$ & $15-3.3$ \\
\hline \multirow[b]{2}{*}{ Green semiconductor laser } & ferrofluid Pure & $0.5-0.3$ & 15- 2.9 \\
\hline & $\begin{array}{l}\text { ferrofluid doped } \\
\text { with copper } \\
\text { nanoparticles } \\
\end{array}$ & $0.5-1$ & $12-2.8$ \\
\hline \multirow[b]{2}{*}{ He-Ne Laser lasers } & ferrofluid Pure & $0.5-0.4$ & $8.5-3$ \\
\hline & $\begin{array}{l}\text { ferrofluid doped } \\
\text { with copper } \\
\text { nanoparticles }\end{array}$ & $1-4$ & $15-9.6$ \\
\hline
\end{tabular}

The figures of the hysteric loop show the variation of the magnetic flux density of the ferromagnetic material with the external magnetic field producing it as this field changes through a complete cycle. From these figures, the area of the loops which are referred to as the amount of energy lost when applying the external magnetic field means there is more energy loss or more work done in the magnetization and demagnetization of the material. The hysteresis loop of the pure ferrofluid magnetic switch with violet, green and $\mathrm{He}-\mathrm{Ne}$ lasers are shown in Figs.3 (a, b, c). 


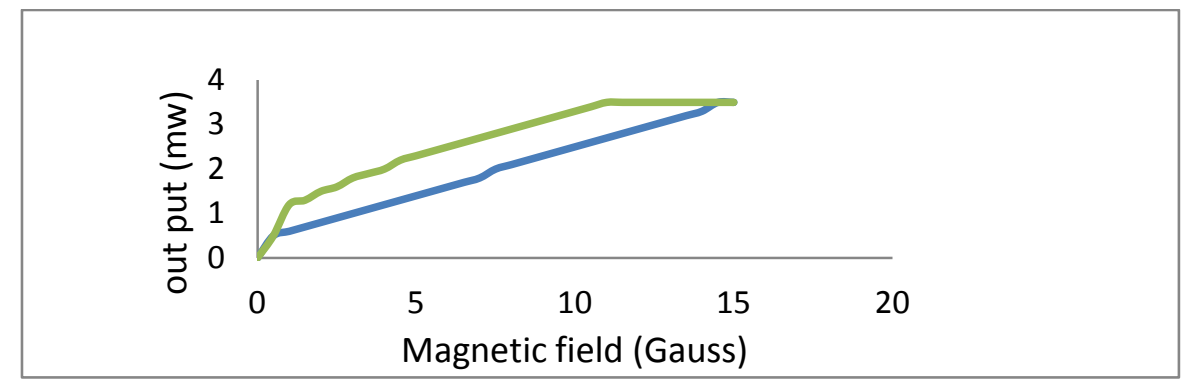

(a)

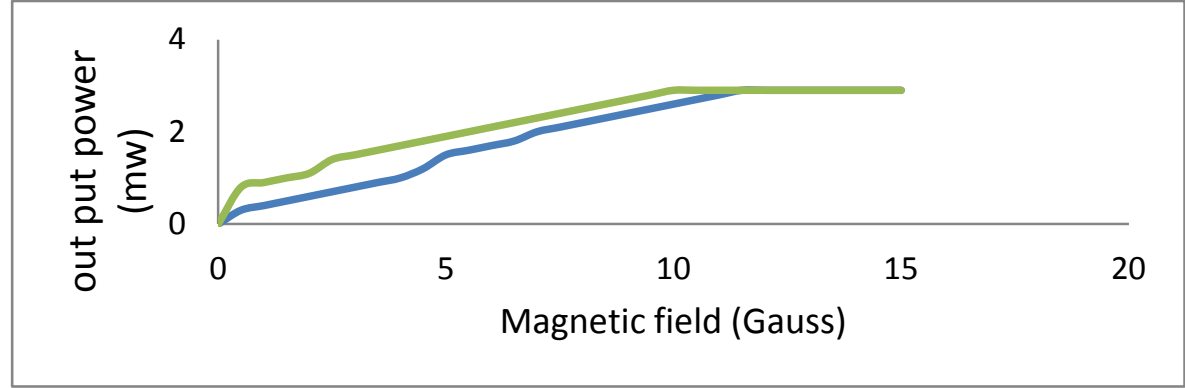

(b)

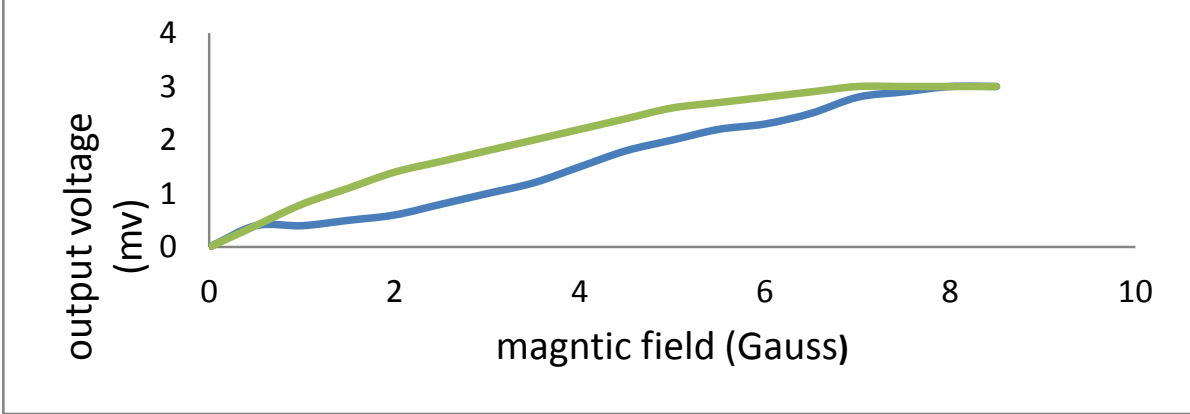

(c)

Fig.3: The hysteresis loop of pure ferrofluid magnetic switch with violet, green and the He-Ne Laser.

The hysteresis loops of the waterbased ferrofluid doped with copper nanoparticles magnetic switch using violet, green, and He-Ne laser sources are shown in Figs. 4 (a, b, c). This doped material showed new physical and chemical properties because of their small size and large surface area, which gave new hysteresis loops of the magnetic switch that is differ from the loops of the magnetic switch without doping. The area of the formed hysterical loop is narrower than that of the pure sample. This is because that the magnetic field does less work on the switch than the pure one and the loss is less than in the pure sample. The narrowest hysterical loop obtained from the use of ferrofluid doped with copper nanoparticles in the manufacturing of the magnetic switch is due to the fact that water is highly polarized and its work is to regulate the nanoparticles in magnetic fluids which reduces the losses in the magnetic switch and reduces the work done on it. When using doping, sensitivity to the magnetic field is not the fastest, but the best for the doped samples. 


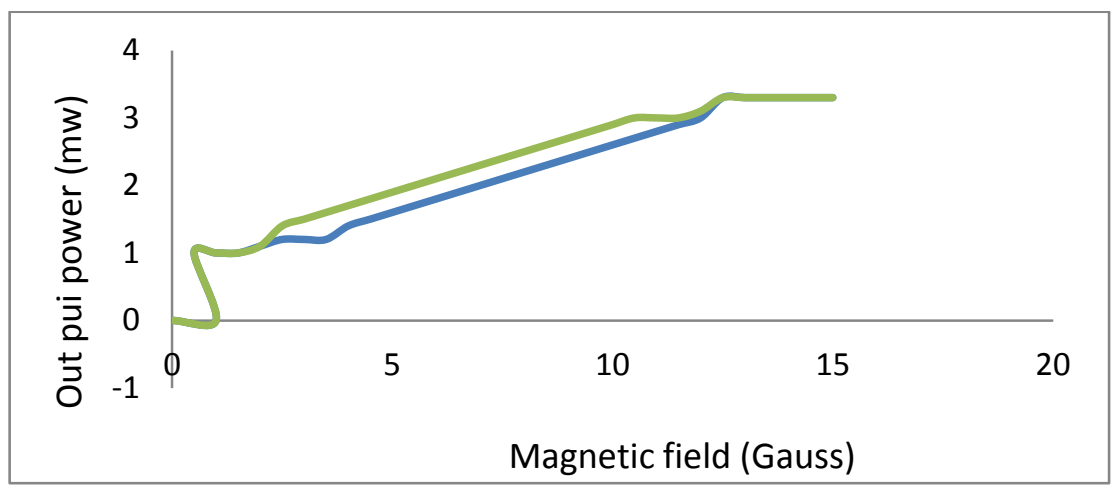

(a)

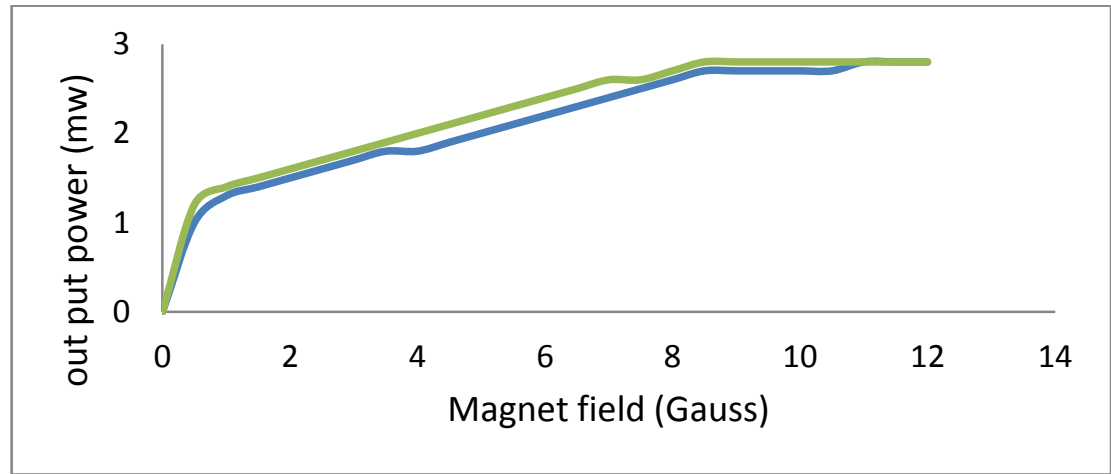

(b)

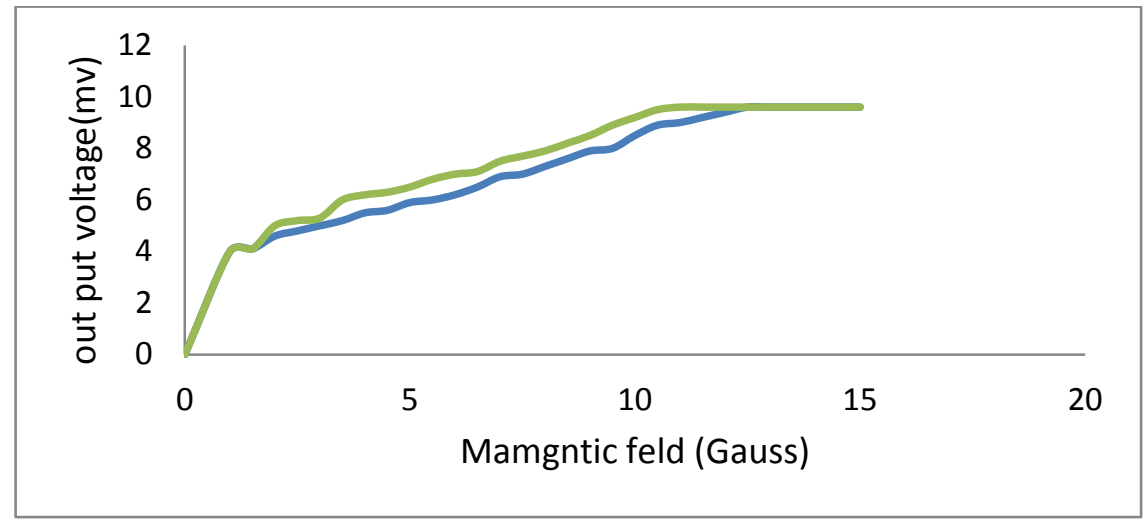

(c)

Fig.4: The hysteresis loop of ferrofluid that is doped with copper nanoparticles using violet semiconductor, green and $\mathrm{He}-\mathrm{Ne}$ Laser.

\section{Conclusions}

Best results for the critical magnetic field and the saturation magnetic field were obtained, for copper nanoparticles doped ferrofluid, when using He-Ne laser. The narrowest hysterical loop was obtained from the use of water based ferrofluid doped with copper nanoparticles $(10 \mathrm{~nm})$ in the manufacturing of the magnetic switch. This is because water is highly polarized it regulates the nanoparticles in the magnetic fluids. This reduces the losses in the magnetic key and reduces the work done on it.

\section{References}

[1] Zaki M. Moutassem "A Theoretical and Experimental Analysis of the Density Separation of Nonmagnetic Materials with the Use of Imposed 
Magnetic Fields", Ph.D. Thesis, University of Toledo, (2016)

[2] J. Popplewell, Physics in Technology, 15, 3 (1984) 150-156.

[3] Thomas A. Franklin, "Ferrofluid Flow Phenomena", Ph.D. Thesis, Massachusetts Institute of Technology, (2003).

[4] C. Scherer, N. Figueiredo, M. Antonio, Brazilian Journal of Physics, 35, 3 (2005) 718-727.

[5] SGE. Lampaert, JW. Spronck, RAJ van Ostayen, Part J: Journal of Engineering Tribology, 232, 1 (2018) 14-25.

[6] Chin Wang and Luogen Deng, Laser Phys. Lett., 11 (2014) 1-13.

[7] Rawa K. Ibrahim AL-Meshahedi "Characterization of Nanoparticles Doped Liquid Crystal as an Optical switch", Ph.D. Thesis, University of Baghdad, (2015).
[8] Rabi N. Hammaudi AL-Waali "Fabrication of Optical Switch based on Ferronematic and Ferrofluids", University of Baghdad, (2018).

[9] Jae-Hee, K. Hyeon-Seok S, YounJea K., Micromachines, 9, 457 (2018) 1-8.

[10] Alvaro R., Tim H. Hermans J., Gabriel C., Lidia P., Miguel A., Elena C, 2nd Symposion on Space Educational Activities, Budapest, Hungary, (2018) 1-5.

[11] Milorad M. Kuraica, Predrag Iskrenović, Marko Perić, Ivan Krstić, Aleksandar S. Nikolić, Chemical Papers, 72, 6 (2018) 1535-1542.

[12] Libo Huang, Torsten Hädrich, Dominik L. Michels, ACM Transactions on Graphics, 38, 4 (2019) 93-1_93-15. 